CHERCHER, REPÉRER, AVANCER

\title{
L'ÉDUCATION PAR LE SPORT FACE AU CHÔMAGE
}

François Le Yondre, Dominique Bodin, Peggy Roussel et Luc Robène

\section{ADRESE/CIRNEF | «Les Sciences de l'éducation - Pour l'Ère nouvelle »}

2010/3 Vol. 43 | pages 37 à 53

ISSN 0755-9593

ISBN 9782918337058

Article disponible en ligne à l'adresse :

https://www.cairn.info/revue-les-sciences-de-l-education-pour-l-erenouvelle-2010-3-page-37.htm

Distribution électronique Cairn.info pour ADRESE/CIRNEF.

(C) ADRESE/CIRNEF. Tous droits réservés pour tous pays.

La reproduction ou représentation de cet article, notamment par photocopie, n'est autorisée que dans les limites des conditions générales d'utilisation du site ou, le cas échéant, des conditions générales de la licence souscrite par votre établissement. Toute autre reproduction ou représentation, en tout ou partie, sous quelque forme et de quelque manière que ce soit, est interdite sauf accord préalable et écrit de l'éditeur, en dehors des cas prévus par la législation en vigueur en France. Il est précisé que son stockage dans une base de données est également interdit. 


\section{L'éducation par le sport face au chômage}

François Le YONDRE*, Dominique BodiN**,

Peggy RousseL ${ }^{* * *}$ et Luc RobèNE ${ }^{* * * *}$

Résumé : Dans quelles conditions le sport peut-il être éducatif pour les chômeurs? Cette question élargit doublement la problématique de l'éducation par le sport généralement cantonnée aux jeunes populations et se réduisant bien souvent à la question des apports de la pratique. Admettant de ne pas connoter positivement l'adjectif, l'article interroge le caractère éducatif du sport au sens où il permet l'incorporation des normes et des valeurs corporelles et relationnelles. Nous avons réalisé quatorze entretiens semi-directifs avec des chômeurs engagés dans un stage de redynamisation par le sport. Sept autres ont été réalisés avec des chômeurs ayant une pratique sportive spontanée. Au sein de ces stages, le sport, présenté comme une activité d'engagement envers soi et autrui, est à la fois le support d'un rappel normatif adressé aux chômeurs dont on soupçonne la déviance volontaire et un espace de conformité favorable à une revalorisation de l'estime de soi.

Mots-clés : Chômeur. Sport. Éducation.

\footnotetext{
* Docteur-ATER, Université de Rennes 2, UFRAPS.

** Professeur, Université de Rennes 2, UFRAPS.

*** Maître de conférences, Université d'Aix-Marseille II.

**** Maître de conférences HDR, Université de Rennes 2, UFRAPS.
} 


\section{Introduction}

La problématique de l'éducation par le sport est généralement appréhendée à partir des actions engagées en direction des jeunes populations des quartiers sensibles. Cette centration est celle des commandes politiques préoccupées par les flambées de violence et imprégnées d'une conception simpliste voire idéologique d'un sport aux vertus pacificatrices. L'article propose une approche extensive de cette problématique. Il s'agit d'une part de déplacer la cible. Bien que l'enfance et l'adolescence soient évidemment les âges auxquels les sciences de l'éducation se destinent prioritairement, il existe cependant des temps de la vie qui rappellent avec force le caractère permanent de la construction de l'individu. En tant que choc biographique, le chômage nous semble être l'un de ces temps. Pour de nombreux chômeurs, il constitue une véritable épreuve (SCHNAPPER, 1994). Nous nous attarderons plus précisément sur les dimensions temporelle et corporelle de cette épreuve. Une forme de dévitalisation du corps et une déstructuration du cadre temporel plongent en effet les chômeurs dans une torpeur générale où la vie sociale est réduite à sa plus légère expression. Mais loin de nous l'idée d'activer l'approche misérabiliste du chômage. Rappelons pour cela l'hétérogénéité des formes du chômage (Freyssinet, 1993) et de ses vécus. Des travaux ont montré par exemple les réinvestissements positifs du chômage (SCHNAPPER, 1994; GrELL, 1985; Roulleau-Berger, 1991; Schehr, 1999). Cependant, il ne s'agit pas non plus de réenchanter la précarité en oubliant la faible représentation quantitative de ces chômeurs qui ne subissent pas l'expérience du chômage. C'est pourquoi nous nous intéresserons exclusivement au chômage total (SCHNAPPER, 1994) qui constitue l'expérience la plus répandue, vécue par tous ceux dont le niveau culturel modeste et la faible insertion sociale empêchent d'en tirer parti.

L'approche extensive de la problématique est par ailleurs inscrite dans la définition de l'adjectif éducatif. Certes, nous retenons le sens classique et individuel en interrogeant le sport comme moyen de faire face à l'expérience déstructurante du chômage total. Mais bien souvent, poser la question du potentiel éducatif du sport revient seulement à dégager le positif que les pratiquants peuvent en tirer en termes d'apprentissage. Nous prenons le parti d'interroger le caractère éducatif du sport au sens où «il permet d'intérioriser les significations sociales, d'incorporer des valeurs et des idéaux non seulement corporels mais également relationnels, d'apprendre la légitimité des principes régulant leurs actions individuelles et collectives. " (LIOTARD, 2004, p. 120). Les deux acceptions du terme se révéleront finalement complémentaires. Lorsqu'il est fait usage du sport pour diffuser les normes communes auprès des chômeurs, ceux-ci y voient une occasion de conformité valorisante. 


\section{Méthodologie}

L'enquête repose principalement sur des entretiens semi-directifs (DEMAZIÈRE, DUBAR, 1997) effectués avec 21 chômeurs sportifs vivant un chômage total, et articulés autour de trois thèmes : le récit du parcours professionnel, le vécu du chômage et les motivations à la pratique sportive et ses effets. Au-delà de ces thèmes nous avons laissé libre cours à la parole des «informateurs» (KAUFMANN, 1996) afin de pouvoir repérer plus facilement l'émergence d'identités narratives (KAUfMANN, 2004). Solliciter le récit de soi attise déjà les stratégies discursives visant à présenter une image de soi cohérente par la réinterprétation d'éventuelles contradictions biographiques. Nous considérons l'entretien comme un moment de construction sociale de la réalité. Le subjectif, lui-même en construction lors de l'entretien quand l'acteur tisse un lien de cohérence au cours de sa narration, est une étape de cette construction si l'on dépasse l'opposition classique subjectif/objectif. "La représentation n'est pas un simple reflet, elle est un moment crucial dans le processus de construction sociale de la réalité.» (KAUFMANN, 1996, p. 60). C'est pourquoi le discours des chômeurs à propos de leurs pratiques sportives est une étape de la construction sociale de la réalité. Le caractère éducatif du sport ne peut être contenu intrinsèquement dans l'activité. Nous émettons l'hypothèse qu'il se construit dans l'interaction entre les politiques de traitement du chômage par le sport et leurs destinataires.

$\mathrm{Au}$ cours de deux stages de redynamisation par le sport pour bénéficiaires du RMI, financés par les conseils généraux, nous avons interrogé quatorze bénéficiaires. Nous avons également rencontré six chômeurs de ce type, et ayant une activité sportive, à la sortie des ANPE. Enfin, nous avons interrogé Eric Fournier, ancien bénéficiaire du RMI ayant réalisé un exploit sportif très médiatisé (tour de France à vélo) afin de mettre en scène sa recherche personnelle d'emploi et faire la lumière sur le chômage des quinquagénaires. En bigarrant les modes de recrutement nous recherchions la diversité du panel et guère sa représentativité.

Les entretiens ont été renforcés par des observations participantes au cours des stages. D'emblée, nuançons l'emploi de la formule «observation participante». Nous n'étions pas chômeurs mais clairement identifiés comme étudiants travaillant sur la pratique sportive. Par conséquent, il est impossible de savoir dans quelle mesure l'intégration apparente dont nous bénéficions était réelle. Ce qui ne signifie cependant pas que leurs comportements et propos en notre présence ou lors des entretiens furent moins réels qu'en notre absence (MAUGER, 1991). L'individu peut être différent mais le rôle que notre présence lui fait endosser n'a pas moins de valeur. Le rapport d'enquête demeure un rapport social. En revanche, 
l'entretien doit permettre un relâchement progressif de ce rôle afin de "produire des effets de vérité»" (KAUFMANN, 1996, p. 69). Or dans le cadre d'une enquête sur la pratique sportive, la participation nous semble une opportunité pour la profondeur de l'entretien qui suit. L'implication physique du chercheur est une sortie de son rôle strict et un engagement favorisant celui de l'informateur. La narration de soi est un risque, une mise en danger de soi au regard d'autrui. Il nous a semblé que nos interviewés acceptaient d'autant plus facilement cette "prise de risque» dès lors que nous étions «avec eux» et mis, nous-mêmes, en danger. D'autant plus que le débridement au cours de la pratique et l'engagement corporel sont souvent perçus comme le dévoilement de son identité réelle. L'association de l'authenticité de l'être à la pratique sportive est fréquemment exprimée à travers ce type d'assertion récurrente : «En sport, on ne peut pas tricher. " L'intérêt de cette implication physique tient également à la présence des sensations corporelles comme thème fort des entretiens. Les connaître par le corps, au-delà du discours, représente un avantage certain.

\section{Le sport face à l'épreuve ${ }_{\text {les chômeurs, il convient de s'arrêter en }}^{\text {Pour }}$ temporelle du chômage amont sur les effets du chômage total. Il est incontestablement une épreuve temporelle. Nonobstant la baisse tendancielle du temps} de travail et la pertinence des thèses sur l'effritement de la centralité de la valeur travail, le temps professionnel demeure celui autour duquel se structurent les rythmes quotidiens et hebdomadaires, «le temps de référence» (DEMAZIÈrE, 2006). Ceci est d'autant plus valable pour les chômeurs interrogés que le chômage total se définit en partie par l'incapacité à relayer le travail au second rang, de le réduire à sa dimension instrumentale. À défaut d'être un temps de liberté à investir comme en situation de chômage inversé, le temps est vide, voire déqualifié pour Éric : «Oui le temps qui passe quand il est pas qualifié. On qualifie normalement nos journées. On donne un emploi du temps, une règle. Là elles sont pas qualifiées.» (Éric, 48 ans). Pour un individu en chômage total, il est difficile de formuler ce qu'il a fait dans la journée. L'absence d'activité chargée de sens est indicible. Le vide est donc également discursif et ne pas être en mesure de dire ce que j'ai fait renvoie à l'incapacité de dire qui je suis, voire de dire que je suis : "Tu te dégoûtes parce qu'en fin de journée, c'est une journée où t'as pas vécu, t’aurais pas existé, ça aurait rien changé. Tu sers à rien, t’as rien fait.» (Julien, 28 ans). Ce temps vide questionne l'existence même de l'individu puisque «toute mon existence dans ce 
monde est ordonnée par ce temps et enveloppée par celui-ci. Ma propre vie est un épisode du courant extérieurement factice du temps. La connaissance de ma mort inévitable rend ce temps fini pour moi. Je ne dispose que d'un certain temps pour réaliser mes projets, et la connaissance de cette contrainte affecte mes attitudes envers ces projets.» (BERGER \& LUCKMANN, 1989, p. 43). Le chômage renverse ce rapport au temps. Celui-ci n'est plus à investir mais à combler. Notons «qu'il existe dans les sociétés contemporaines, deux cultures du temps parfaitement antagoniques.» (KAUFMANN, 2004, p. 231). Certains entretiennent de multiples projets que leurs ressources rendent plausibles et manquent de temps pour les réaliser alors que des chômeurs souffrent de trop de temps sans que leurs ressources ne leur permettent de se projeter. L'existence même de l'individu est remise en cause au point que le chômage est parfois assimilé à une mort sociale. La plupart des chômeurs interrogés ont tenté d'échapper au temps qui passe par le sommeil prolongé : «Les journées sont courtes. À un moment je dormais vachement. Tu rentres dans un système où tu dors 18 heures sur 24 quoi.» (Nathalie, 28 ans). C'est alors la vie ellemême qui est réduite à sa plus légère expression. De surcroît, «l'horloge et le calendrier assurent, en effet, que je suis un homme de mon temps. C'est seulement à l'intérieur de cette structure temporelle que la vie quotidienne garde, à mes yeux, son accent de réalité.» (BERGER \& LUCKMANN, 1989, p. 43). C'est pourquoi il convient de «considérer le temps des chômeurs comme le produit d'interactions avec autrui plutôt que comme un point de vue interne à l'individu" (Demazière, 2005, p. 3). Le décalage avec le rythme collectif donne l'impression d'un éloignement de la réalité collective et renforce le sentiment d'exclusion. Le crédit de réalité de sa propre existence en est également réduit : «Voilà et depuis que je suis sans travail euh, j’ai un peu perdu disons le sens de la réalité du quotidien.» (Patrick, 50 ans).

En compensation, le sport semble donner un élan se poursuivant au-delà du temps de pratique : "Ce que j'aime c'est aller dès le matin, tu commences ta journée par ça et ça te met sur de bons rails pour attaquer, ca me donne de l'énergie, je me sens bien dans ma peau.»(Denis, 36 ans). Denis revalorise son estime de soi en parvenant à qualifier son temps, ce qui l'incite à poursuivre dans ce sens afin de faire correspondre l'avenir immédiat de son agenda à cette image positive de lui-même. Ce qui fonctionne ici à une échelle quotidienne pour Denis semble viable au-delà pour ceux qui sont engagés dans un stage : «On peut dire que c'est un jour oui et un jour non. Le sport c'est pas le lundi mais le mardi, pas le mercredi mais le jeudi. Et c'est vrai qu'au départ ça a été dur le réveil à 7 h30 parce que quand on a rien à faire, on se lève quand on se lève mais finalement de me lever un jour sur deux, ben quand c'est pas le jour je suis quand même réveillé à $7 \mathrm{~h} 30$.» (Alice, 45 ans). Alice expose ici sa stratégie mentale visant à qualifier son 
temps et à résister à la décomposition du cadre temporel qui entraîne avec elle le sentiment d'utilité voire d'existence sociale. Elle s'appuie sur les deux jours de pratique hebdomadaires (les jours oui) afin de structurer l'ensemble du cadre temporel de la semaine. Ainsi, les jours de non pratique (les jours non) semblent insérés entre deux jours de pratique. En réalité, seul le mercredi est dans ce cas alors que les autres jours sont insérés entre un jour de week-end et un jour de sport. Pour Alice, ce qui importe est de ne pas avoir en vue deux jours non qualifiés pendant lesquels il est convenu de travailler. Le samedi et le dimanche ne sont ni des jours «oui» ni des jours «non"; ils sont des jours où les chômeurs ne sont pas distingués par l'inactivité puisqu'elle est le lot de chacun.

Lactivité sportive, programmée dans un cadre temporel, semble être un moyen de surmonter l'épreuve temporelle du chômage. Mais rien n'indique qu'une autre activité ne puisse en faire autant. Il convient de dégager les spécificités du sport et les conditions qui permettent d'en faire un support privilégié de reconstruction individuelle face à l'épreuve du chômage.

\section{Le sport pour un agenda du corps}

La dimension temporelle du chômage total en fait également une épreuve corporelle. Ceci intéresse les propriétés éducatives du sport car l'action motrice constitue la spécificité première du sport. Aussi la structure temporelle commune devient généralement intériorisée au point de l'incorporation. Il est dit en effet des habitudes qu' elles sont relativement incorporées. Cette incorporation libère l'action. Le simple fait que les gestes soient à ce point intériorisés permet à l'acteur d'échapper à une permanence de la réflexivité potentiellement envahissante (BERGER \& LUCKMANN, 1989). Ceci dit, lorsque l'on a admis que l'habitude est un schème incorporé, il devient aussi légitime qu'apparemment candide de se demander dans quelle partie du corps elle vient se loger. En réalité, il faut, pour envisager l'incorporation selon Kaufmann (2001), cultiver une conception du corps qui dépasse le simple corps biologique. «L'habitude n'est pas dans ce corps là, elle est dans le corps élargi qui s'inscrit dans l'univers social.» Il propose pour définir ce corps élargi trois extensions dont la première est éclairante ici. "L'habitude n'est pas inscrite à l'intérieur du corps biologique mais dans les lignes de ses mouvements et déplacements, dans les gestes.» (KAUFMANN, 2001, p. 173). C'est donc dans le mouvement que le corps intègre les habitudes et qu'il crée de l'évidence et du sens pour l'acteur. Ce sont les mouvements et les rythmes habituels du corps qui apaisent l'individu en le déchargeant de sa réflexivité, libérant à 
nouveau l'action. Or l'activité professionnelle définit les rythmes corporels au quotidien et se répète suffisamment pour que l'incorporation soit effective. La perte de l'emploi est un arrêt brutal du mouvement dans lequel les habitudes et les évidences ne peuvent plus se loger. L'inertie soudaine des corps des chômeurs donne lieu à une béance dans laquelle la réflexivité de l'individu est happée, s'engouffre et cherche à quoi se raccrocher. Cette inertie des corps au chômage engendre un réel déficit de sens qui semble expliquer partiellement le malaise exprimé par les chômeurs : "J'ai besoin de bouger, d'être actif, de donner du sens à ma vie. J'étais quasiment emprisonné dans cette période là. [...]. C’est comme une avalanche qu'entraîne plein de choses. Des horaires de sommeil irréguliers, on mange mal, on dort tard, ça ne veut plus rien dire la vie.» (Ramine, 44 ans). Le sport programmé selon une régularité réinscrit le corps dans un flux de gestes. Il donne accès aux sensations de fatigue qui sont les traces corporelles d'une activité alors que l'inactivité dévitalise le corps au point de l'oublier parfois : «Au bout d'un moment, on oublie la conscience de son corps. On oublie qu'on a un corps qui a besoin qu'on s'en occupe. Et c'est par l'hygiène alimentaire et aussi en faisant du sport, l'hygiène corporelle en se lavant. Je pense que dans le processus de désolidarisation, de dépression, on oublie tout ça." (Ramine, 44 ans). La pratique sportive procure à nouveau les sensations d'une existence après un oubli voire une mort du corps condamné par son inutilité sociale. Elle procure à Céline le sentiment d'une renaissance qui ne semble pas uniquement corporelle : «Tu sens qu'il y a tout qui bouge. Pour moi il était mort le corps. Et quand tu fais du sport, tu sens le sang bouger à l'intérieur, ça revit. Je me sens plus forte, j'ai l'impression que c'est de la vie qu'il y a en moi. J'ai carrément l'impression d'être une autre personne. Je me sens plus légère. Même si j'ai mal aux jambes, j'arrive à parler aux autres.» (Céline, 28 ans). La renaissance perçue par Céline semble effectivement dépasser le temps de la pratique. Dans une situation professionnelle conforme, le corps à travers les sensations physiques, s'inscrit dans un calendrier, une sorte d'agenda du corps. En revanche, le chômage prolongé le plonge dans une aphasie qui participe à dissoudre la notion de temps. En compensation de l'activité professionnelle, le sport donne l'accès à cette espèce d'agenda du corps. Il permet de ressentir les variations de son état corporel et de les agencer dans le temps : «si je viens au sport c'est que je sais que je vais me dépenser sinon je viendrais pas. Je sens que j'en ai besoin, là on en fait deux fois par semaine, le mardi et le jeudi donc avec le week-end ça fait à peu près tous les deux trois jours et ça me permet d'évacuer les tensions accumulées en trois jours.» (Céline, 28 ans). La construction de cet agenda apparaît très clairement ici. Pour le chômeur, la remise en mouvement du corps permet d'échapper au vide que l'inertie attise. Ce vide 
n'est pas seulement celui de l'agenda. Il est plus globalement ontologique et renvoie au sentiment d'inutilité, à la constance des sensations corporelles.

Il est fréquent de lire que c'est en «dé-routinisant » que le sport permet d'apaiser l'individu (SEmpé, Gendron \& Bodin, 2007). Notre constat est à la fois très proche et totalement contraire. Car, en effet, la décharge d'une énergie accumulée semble salutaire pour les chômeurs mais elle entraîne en revanche la reconstruction de ce que nous appelons un agenda du corps inverse à la logique d'une "déroutinisation". Le sens commun envisage la routine comme un poids mais, proche de l'habitude, elle apaise l'acteur et libère l'action (BERGER \& LUCKMANN, 1989). L'absence d'agenda se trouve d'ailleurs au fondement de l'épreuve du chômage. La pratique sportive programmée et régulière permet d'insérer dans une structure temporelle des variations de l'état corporel. D'une certaine façon, ces mouvements du corps sont créateurs de sens pour le chômeur. Ils se présentent comme un rempart à réflexivité happée par l'inertie. Ceci est d'autant plus valable lorsque le rythme de la pratique sportive se rapproche du rythme de l'activité professionnelle de ceux qui ont un emploi : "Maintenant j’y vais et je me donne bien quoi et je peux te dire que j'arrive le soir et je sens bien dans les jambes que j'ai pas glandé dans la journée.». (Myriam, 43 ans). Les sensations corporelles (ici la fatigue) insérées dans la structure temporelle quotidienne commune (ici la fin de journée) permettent à Myriam de négocier avec elle-même une identité pour soi en tenant pour référence normative le temps professionnel. Les traces de l'effort, au moment même où ceux qui ont un emploi rentrent également chez eux, réinscrivent son corps dans une temporalité commune.

\section{Du contrat sportif au contrat social}

Au chômage total, l'individu appréhende le sport comme une futilité. Nous l'avons constaté lorsqu’à la sortie des ANPE, nous interrogions les chômeurs sur leur éventuelle pratique sportive. Beaucoup ont haussé les épaules avant de s'en aller pour signifier le caractère saugrenu de la question : estce vraiment sérieux de faire du sport quand on est au chômage? Avant le stage, Julien a fait l'expérience du chômeur sortant de son rôle en faisant du sport : «Tu vas tout seul courir, mais au bout de quelque temps c'est bon quoi, terminé. Tu te dis à quoi ça sert, qu'est que je fous là à courir dans le vent, comme un con un peu quoi. En plus t'as pris du poids, t'avances pas, t'es ridicule [...] Tu te dis j'ai rien à foutre là pendant que tout le monde bosse.» (Julien, 28 ans). De la même façon, Denis pratique du sport pour son bien-être mais paradoxalement ne 
s'autorise pas à pratiquer lorsque sa situation s'aggrave : "Je suis mentalement pas disposé quand mes ressources financières sont limitées. Elles le sont tout le temps mais quand la situation atteint les extrêmes comme actuellement, c'est pas évident. C'est vrai ça coûte rien. Ça reste quand même secondaire et on pense moins aux choses secondaires dans ce genre de posture.» (Denis, 36 ans). La pratique sportive spontanée (en dehors d'un stage) apparaît fragile pour deux raisons. Elle correspond à une sortie du rôle communément attribué au chômeur et l'expérience de Julien le montre clairement. Aussi, le temps dissout du chômage laisse la possibilité de reporter constamment la pratique. D'après Ramine, à défaut d'être motivé voire obligé par quelqu'un, le chômeur doit réaliser un acte héroïque pour décider d'aller faire du sport et ainsi briser le cercle vicieux de la dévitalisation du corps et de l'autodépréciation : "Chaque pas vous coûte, faire des efforts physiques vous coûte. Et comme c'est un cercle fermé, pour le briser, il faut une action hérö̈que. Parce qu'on ne s'en rend même pas compte de tout ça. On a pas une vision extérieure, une vision neutre et bienveillante par rapport à soi. Au contraire on a une image de soi qu'est pas appréciable.» (Ramine, 44 ans). Éric a réalisé cet acte héroïque en mettant en scène son exclusion et sa recherche d'emploi en parcourant la France à vélo. Mais il prétend être allé chercher chez les autres sa propre motivation «en se verrouillant» dit-il. Les articles de presse annonçant son projet ne lui ont plus laissé le choix en suscitant des attentes chez un entourage soudainement élargi. La réalisation de son exploit est alors devenu obligatoire, ce qui lui a permis de le mener à bien : «Je me suis volontairement verrouillé. J'étais content quand cet article est paru. C'est un verrou. Parce que j'ai plus le choix après. Tout le monde savait que je partais. J'étais obligé. J'ai eu deux trois jours d'angoisse après l'article. Je me suis dit t'es complètement taré. Puis je me suis dit bon maintenant, t'as que ça à faire, y aller. Et sans verrou, j'aurais peut être fait marche arrière.» (Éric, 48 ans). Le verrou d'Éric est l'engagement envers autrui. En formulant une promesse, il s'expose à la déception des autres significatifs et à la dégradation de son identité pour autrui et de son estime de soi. Selon des formes variées, cet engagement s'avère un moteur de l'action sportive. Lors d'une simple course à pied, les attentes d'autrui stimulent l'individu soucieux de se préserver d'une dépréciation identitaire : "Quand on court à plusieurs c'est différent y'a l'entraînement du groupe quoi, on continue avec tout le monde." (Jean-Jacques, 56 ans). Notons en parallèle que la situation de ces chômeurs ayant basculé dans l'assistanat, se caractérise par un déséquilibre du rapport contractuel légitimant l'appartenance de chaque membre à la société. Cet équilibre tient dans l'unité dialectique du droit et du devoir. Or celle-ci est rompue lorsque «la société me verse une allocation sans rien me demander, donc sans me conférer de droit 
sur elle.» (GoRZ, 1988, p. 254). Le sentiment d'être une charge, de profiter d'un système économique sans y contribuer prend probablement aussi sa source dans l'existence d'un traitement politique individualisé du chômage qui tend à faire endosser la responsabilité aux chômeurs (PIERRU, 2005). Les services sociaux font certainement usage de cette norme du contrat auprès des bénéficiaires du RMI afin de réinstaurer un rapport contractuel et de compenser le déséquilibre perçu entre les droits et devoirs du bénéficiaire. Les discours et pratiques des services sociaux semblent favoriser l'intériorisation de cette norme du contrat. Or les associations, financées par leurs conseils généraux, qui proposent ces stages de redynamisation par le sport, relayent ce discours et font du sport une activité d'engagement envers soi et envers autrui. À défaut de pouvoir honorer ses devoirs qui légitiment la protection dont ils bénéficient, le sport devient pour les chômeurs une occasion de prouver leur aptitude à respecter un engagement. Il s'agit bien de lutter contre le stigmate en mettant à distance la figure du faux chômeur dont l'imposture serait de profiter de droits sans en assumer les devoirs corollaires. Franck a clairement intériorisé cette conception d'un sport chargé de plaisirs qui nécessite des concessions envers le collectif : Le sport c'est pas toujours facile. Moi, je jouais milieu et je me retrouve à l'arrière. Et pourtant j'aime pas mais je le fais quand même. C'est aussi ça le sport, et le boulot c'est un peu pareil. Y'a des choses qu'on va te demander au travail que t'aimes pas et que tu seras obligé de faire. Tout est lié là dedans et pour moi le chômeur qui veut vraiment s'en sortir et qui fait du sport, il s'en sort. (Franck, 36 ans). Le parallèle établi entre le sport et le travail, qui requièrent tous deux cette aptitude à se conformer aux exigences du collectif, est révélateur. Il prouve d'une part que l'aptitude à l'abnégation au travail est suggérée lorsqu'elle est démontrée dans un cadre sportif. Le sport semble une sphère plausible de négociation identitaire au-delà du temps de pratique. Plus largement, la continuité de cette norme qui traverse les mondes sociaux (ici les mondes sociaux du travail et du sport) indique sa prégnance générale. Par ailleurs, Elias l'avait déjà présentée comme un principe fondamental de tout groupe social : «On ne saurait recevoir l'aval du groupe sans se plier à ses normes. Toute déviance, réelle ou supposée, se solde par une perte du pouvoir et un abaissement de son statut.» (ELIAS \& SCOTSON, 1997, p. 57). Ce principe se situe au fondement de la stigmatisation des bénéficiaires du RMI. Présenté aux chômeurs comme un espace d'équilibres entre des bienfaits (plaisir, santé) consécutifs à des obligations tenues envers soi et autrui (engagement physique jusqu'à la douleur, entraide, ponctualité), le sport devient donc une activité de rééquilibrage entre droits et devoirs. Mais le sport devient également un support éducatif au sens où il diffuse les valeurs dominantes et naturalise le rapport, en l'occurrence contractuel, à autrui. Lorsqu'on parvient à ne pas connoter «positivement» l'adjectif éducatif, il devient possible d'enregistrer 
l'action éducative du sport qui transmet et légitime les principes régulateurs des rapports sociaux. Ainsi lorsque nous demandons à Alice si elle envisage de retravailler, elle présente son investissement dans le stage de sport comme un premier pas et assure sa fidélité à son engagement contractuel, se défendant par là-même du soupçon permanent qui pèse sur les chômeurs; celui de jouir de droits sans devoirs corollaires : «Y'en a qui ont besoin de barrières. Moi j’ai pas besoin qu'on m'en mette, $\mathrm{j}$ 'ai signé un contrat comme quoi je devais venir à chaque séance, et je suis là même quand on est pas obligés.» (Alice, 45 ans). Ce contrat est signé entre le bénéficiaire du RMI et son référent social. Il est en réalité une condition indispensable de la participation au stage; il est donc clairement simulé. Or, nous constatons que l'obligation envers autrui a fréquemment été réinterprétée comme un engagement formulé envers soi-même. Lorsque l'action sportive est accomplie, il résulte un sentiment de fierté d'avoir su s'obliger à l'accomplir : «Pour moi c'est un petit défi, c'est positif surtout par rapport au comportement que j'avais les cinq années précédentes où je me laissais aller. Ça veut dire peut-être que je me laisse moins aller.» (Patrick, 50 ans). Culpabilisante en amont, cette norme du contrat, une fois réinterprétée, semble constituer un support à la revalorisation de l'estime de soi. Le sport est donc éducatif aux deux sens du terme que nous lui prêtions en introduction. D'abord parce qu'il transmet la norme de l'individu responsable à travers le principe du contrat et de l'engagement respecté bien qu'il le déstabilise simultanément puisque la posture du bénéficiaire du RMI se caractérise par une déviance à cette norme. En revanche, lorsque le contrat est respecté et que l'action sportive est réinterprétée par le chômeur sportif comme un engagement autonome envers soi-même, elle devient éducative au deuxième sens plus commun du terme. Autrement dit, elle permet au chômeur de s'ouvrir à une forme de revalorisation de l'estime de soi : «Je veux me fixer des objectifs et les atteindre, en tout cas, marracher pour les atteindre et être fier de moi. Parce que là vu la situation, je peux pas être fier de moi C'est pour ça que ce matin, je voulais faire des 500 avec le chrono. (Julien, 28 ans).

\section{Corps sales de chômeurs et corps beaux de sportifs}

Si l'action motrice et la dépense physique sont des éléments nécessaires de définition du sport, ils n'en sont pas pour autant suffisants. La promenade ou la simple marche utilitaire peuvent-elles être considérées comme des actions sportives? Disons le clairement : non. L'action motrice, lorsqu'elle n'est pas réalisée comme une fin en soi, ne peut être sportive. Ainsi, l'expression populaire "c'est du sport» nous induit-elle en erreur en ne désignant 
que l'acte moteur. Le sport est une activité sociale clairement déterminée et valorisée au point de devenir «un modèle global» (EHrENBERG, 1991). Cette sublimation n'en fait-elle pas un levier identitaire pour les chômeurs au-delà de la simple dimension physique? En surface, les discours que nous avons relevés désignent exclusivement l'engagement du corps comme seul objectif de leur pratique sportive. Pourtant, la production de cet effort demeure clairement une contrainte sans intérêt lorsqu'elle prend cours en dehors d'un cadre sportif. Lors d'un entretien, Patrick, devenu obèse en cinq ans de chômage, nous livre que sa priorité est de retrouver "la réalité de l'effort», considérant que sa santé est en danger. Pourtant, il affirme par ailleurs éviter les efforts quotidiens tels que le passage d'un grand escalier à proximité de sa résidence. Il concède volontiers l'effort sportif de la course à pied en revanche. La contradiction indique plusieurs choses. D'une part, nous l'avons vu, le temps sportif est un temps social à part entière qui participe à la reconstruction d'une temporalité chez le chômeur. Au-delà, il faut voir que le corps dans l'effort est l'objet de perceptions bien distinctes selon qu'il est inséré ou pas dans un cadre sportif. Au quotidien, Patrick souffre des regards que son corps dans l'effort suscite : «Par rapport à ma corpulence, dans le métro quand j'ai des difficultés, je me sens humilié, pas à l'aise, je transpire alors je descends à la première station et je continue à pied. [...]. Les gens poussent. Ils doivent penser que je prends trop de place.» (Patrick, 50 ans). Que l'obésité de Patrick fasse l'objet d'une stigmatisation perçue n'est pas surprenant. Les personnes souffrant de surcharge pondérale ou d'obésité sont "victimes de stéréotypes négatifs et très puissants comme la mauvaise santé mentale, le manque de confiance en soi ou encore l'absence de volonté.» (AMADIEU, 2002, p. 139). Nous constations pourtant à ses côtés que mettre en scène son corps dans l'effort dans un cadre sportif n'a pas suscité chez lui d'appréhension de la sorte. Le contexte sportif semble rendre acceptable la confrontation des corps à l'effort et à la douleur. Et le constat demeure un euphémisme si l'on considère le spectacle sacré des corps souffrant des sportifs. Le marathonien agonisant sur la ligne d'arrivée suscite tant d'émotions et de respect quand celui qui vainc froidement et trop aisément est parfois déshumanisé si ce n'est soupçonné de tricherie. À l'inverse, le corps qui souffre au quotidien est honteux, sale et repoussant. Il convient de se demander pourquoi la perception des formes des corps, de leurs odeurs ou encore de certaines de leurs substances telle que la sueur est à ce point circonstanciée. «Le sport symbolise et promeut l'image de l'individu autonome, gérant aussi bien sa santé, son apparence physique que son implication dans sa vie personnelle comme l'entrepreneur de sa propre existence.» (EHRENBERG, 1991, p. 183). À l'inverse, le corps obèse souffrant d'une posture quotidienne est perçu comme le signe d'une non-maitrise de soi. Tout comme les 
chômeurs sont enjoints par des politiques d'activation (FRANSSEN, 2006) à entreprendre leurs projets de réinsertion, les individus souffrant d'obésité subissent les injonctions formelles (spots publicitaires) ou informelles à maitriser leurs corps. Notons que cinq chômeurs sur les 21 interrogés souffrent d'obésité. Le corps des chômeurs qui portent les signes de l'inactivité est déviant et perçu comme un corps sale car non entretenu : «Elle rentre le soir et moi ça fait depuis le début de la journée que je suis là. J'ai pas bougé. Et ça se voit quand quelqu'un a rien foutu de la journée, ça se sent même. Un mec qui glande toute la journée devant la télé ça se voit direct.» (Julien, 28 ans). De la même façon, le corps transpirant dans l'effort sportif est accepté voire valorisé, car il atteste d'un entretien corporel durant lequel la souffrance est volontaire. Le temps sportif est un temps de sculpture physique, mentale et morale de soi. Il permet la mise à distance des manifestations corporelles lors des interactions quotidiennes, c'est-à-dire hors des temps de pratique sportive. Notre analyse semble réactiver la thèse d'Elias (1998) pour qui le sport, en tant que parenthèse de débridement et d'intériorisation des mécanismes d'autocontrainte, participe au processus de civilisation. Le sport est à nouveau éducatif dès lors qu'il permet aux chômeurs d'intérioriser les normes dominantes. En l'occurrence, la norme de l'individu autonome et responsable est si bien représentée par le sport qu'il en est un vecteur puissant auprès de ceux que l'on stigmatise comme assistés et dépendants, donc déviants. À ce sujet, les propos d'Eric, ancien bénéficiaire du RMI ayant mis en scène sa recherche d'emploi et la situation des chômeurs quinquagénaires par un exploit sportif, sont éloquents : «L'action sportive c'est une espèce d'égosophie, c'est un nouveau mot là. Pas d'égocentrisme, ni d'égoïsme, c'est le fait de s'occuper de soi. C'est dire merde, je vais prendre du temps pour moi et cette action je la fais pour moi.» (Éric, 48 ans). Par ailleurs, Éric prônera avec insistance ce qu'il nomme «la pratique de soi». L'injonction généralisée à s'entretenir est largement diffusée par le sport auprès de ceux dont on soupçonne des choix de vie contraires en dépit des déterminants globaux du chômage. Ici encore le sport est doublement éducatif conformément aux deux acceptions du terme que nous proposions dès l'introduction. Il inculque les valeurs dominantes aux déviants qui par là même voient dans la pratique sportive l'occasion d'une conformité valorisante.

\section{Conclusion}

Le sport, tel qu'il est présenté par les institutions et reçu par les chômeurs, se révèle donc doublement éducatif. Il est d'abord proposé sous forme de stage et selon une 
temporalité proche du temps de référence qui permet aux chômeurs d'accéder à l'agenda du corps par un agencement temporel des variations des états corporels. Celui-ci comble un vide ontologique happé par l'inertie. Disons plus trivialement que le sport «(re) routinise» les chômeurs. En comparaison, les pratiques spontanées sont irrégulières et ne permettent pas de rompre la dissolution du temps. D'autre part, en l'absence de partenaires et de contraintes externes, le sport est vécu comme une sortie du rôle de chômeur.

Ensuite, le stage s'inscrit dans le cadre d'un contrat entre le référent social et le chômeur stagiaire. La conception d'un sport fait d'engagements réciproques semble activée en compensation d'un déséquilibre entre les droits et devoirs qui marquent la condition de l'assisté social. Si le sport est éducatif au sens où il diffuse auprès des chômeurs ce principe fondamental des rapports sociaux, il en devient clairement un moyen de contrôle social d'individus dont on soupçonne ostensiblement (PIERRU, 2005) la déviance intentionnelle malgré les déterminants globaux. Malgré cela, le rapport contractuel contraint est réinterprété $a$ posteriori par le chômeur comme un défi lancé à soi-même et relevé de façon autonome. Parallèlement au rappel normatif qui leur est fait, le sport devient également pour les chômeurs une occasion de conformité valorisante.

Permettons-nous toutefois une réserve quant à la pérennité de ces effets au vu de la durée limitée du stage et surtout au regard d'éventuelles conséquences inverses de l'"entre soi». Celui-ci ne serait-il pas susceptible de renforcer une identité de déviant ou de favoriser le repli sur soi déjà constaté chez nombre de chômeurs? Notons simplement que la question se pose également à propos d'activités sportives proposées aux jeunes de quartiers sensibles qui seraient, par le biais de cet "entre soi», propres à favoriser le repli communautaire. (BODIN, ROBËNE, HÉas \& Le Yondre, 2007). 


\section{Bibliographie}

AMADIEU J.-F. Le poids des apparences, beauté, amour et gloire. Paris : Odile Jacob, 2002.

Berger P. \& LuckMann t. La construction sociale de la réalité. Paris : Méridiens Klincksieck, 1989.

Bodin D., Robène L., Heas S. \& Le Yondre F. Le sport dans la cité : approche critique d'un traitement préventif de la violence. International Journal on Violence and School, 2007, 4.

Demazière D. \& Dubar C. Analyser les entretiens biographiques. Paris : Nathan, 1997.

Demazière D. Le chômage comme épreuve temporelle. In : Thoemmes J. \& DE TERSSAC G. (Ed.). Les temporalités sociales : repères méthodologiques. Toulouse : Octarès, 2006, pp. 121-132.

ÉLIAS N. \& SCOTSON J. Logiques de l'exclusion. Enquête sociologique au cour des problèmes d'une communauté. Paris : Fayard, 1997.

ÉLIAS N. \& DunNING E. Sport et civilisation. La violence maîtrisée. Paris : Fayard, 1998.

Ehrenberg A. Le culte de la performance. Paris : Calmann-Lévy, 1991.

FrANSSEN A. L'État social actif et la nouvelle fabrique du sujet. In : AsTIER I. \& Duvoux N. (Ed.). La société biographique : une injonction à vivre dignement, Paris : L'Harmattan, 2006.

FreYsSinet J. Le chômage. Paris : La découverte, 1993.

Gorz A. Métamorphoses du travail. Quête de sens. Critique de la raison économique. Paris : Galilée, 1988.

GRELL P. Étude du chômage et de ses conséquences : les catégories sociales touchées par le non-travail. Histoires de vie et modes de débrouillardise. Montréal : Groupe d'analyse des politiques sociales, 1985.

KaUfMANN J.-C. L'entretien compréhensif. Paris : Nathan, 1996.

Kaufmann J.-C. Ego, pour une sociologie de l'individu. Paris : Nathan, 2001. 
Les Sciences de l'éducation - Pour l'Ère nouvelle, vol. 43, n 3, 2010

KaUfmann J.-C. Linvention de soi. Une théorie de lidentité. Paris : Nathan, 2004.

LiotaRd P. L'éthique sportive : une morale de la soumission? In : ATTALi M. (Ed.). Le sport et ses valeurs. Paris : La dispute, 2004, pp.117-154.

MaUger G. Enquêter en milieu populaire. Genèses, 1991, nº 6, pp. 125-143.

PIERRU E. Guerre au chômage ou guerre aux chômeurs. Bellecombe-en-bauges : Éditions du croquant, 2005.

ROUlleAu-BERGER L. Jeunesses et cultures de l'aléatoire : de l'emploi précaire à la socialisation professionnelle. Lyon, rapport GLYSI $\mathrm{n}^{\circ} 1 / 91$, Convention ADRESS/MIRE n 348/89, 1991.

SCHEHR S. La vie quotidienne des chômeurs. Paris : PUF, 1999.

SCHNAPPER D. L'épreuve du chômage. Paris : Gallimard, 1994.

Sempe G., Gendron D. \& Bodin D. Le corps sportif en détention : entre contraintes et libération. Corps, 2007, n 2, pp. 55-60.

\title{
Education of the unemployed through sport
}

\begin{abstract}
This article considers the conditions under which sport can meet the educational needs of the unemployed. This question extends the issues of sports education by addressing the needs of older groups and extending beyond questions of the contribution of practice. Despite possible negative connotations, the article questions the educational character of the sport in the sense where it allows the incorporation of the physical standards and values. We carried out fourteen semistructured interviews with unemployed learners engaged in a reorientation course of through sports training. Seven other interviews were carried out with unemployed already involved in the practice of sport. Within these training courses, sport was presented as an activity of self-actualisation and engagement with others. At the same time, a normative recall addressed to the unemployed explored aspects of voluntary in relation to a potential increase in self-esteem.
\end{abstract}

Key Words: Unemployed people. Sport. Education. 


\section{La educación por el deporte ante el desempleo}

Resumen : ¿En qué condiciones el deporte puede ser educativo para los parados? esta cuestión amplía doblemente la problemática de la educación por el deporte generalmente limitada a los jóvenes y que se reduce a menudo a la cuestión de las contribuciones de la práctica. Admitiendo no connotar positivamente el adjetivo, el artículo plantea el carácter educativo del deporte en cuanto que permite la incorporación de las normas y valores corporales y relacionales. Hemos realizado catorce entrevistas semidirectivas con parados participantes en un cursillo de redinamización por el deporte. Se realizaron otras siete con parados que tenían una práctica deportiva espontánea. En estos cursillos, el deporte presentado como una actividad de compromiso hacia sí y hacia los demás, es el apoyo a la vez de un recordatorio normativo dirigido a los parados de los que se sospecha el desvío social voluntario y un espacio de conformidad favorable a una revalorización de la autoestima.

Palabras claves : Parados. Deporte. Educación. 